\section{Efeitos do treinamento de força sobre os níveis de IGFl e de força muscular nas fases neurogência e miogênica de idosas}

\author{
The effects of strength training on the levels of IGF1 levels and 0 \\ $\mathrm{f}$ muscle strength in neurogenic and miogenic phases of elderly women
}

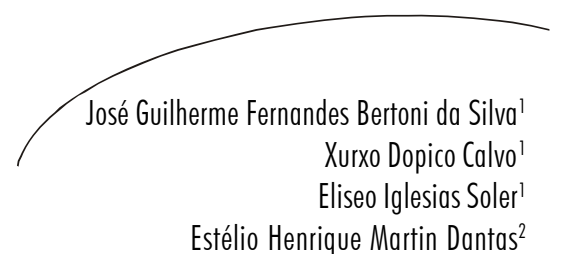

Estélio Henrique Martin Dantas²

\section{Resumo}

O presente estudo teve por objetivo avaliar os efeitos do treinamento de força sobre os níveis basais de IGF1 e de força muscular nas fases neurogência e miogênica de idosas sedentárias. A amostra foi constituída de 24 idosas voluntárias, subdivididas, randomicamente, em dois grupos: grupo experimental (GE, $\mathrm{n}=13 ; 65,62 \pm 5,36$ anos) e grupo controle (GC, $\mathrm{n}=11 ; 71,45 \pm 5,72$ anos). Foi utilizado o protocolo de uma repetição máxima (BAECHLE \& GROVES, 1992) para avaliação da força muscular máxima e de Quimiluminescência - IMMULITE - DPC MED LAB, para o IGF1. Foram utilizados o teste de Kruskal-Wallis (GE, em relação aos três momentos) seguido das comparações múltiplas de Dunn; o teste de Wilcoxon (GC, em relação aos dois momentos) e o teste de Mann-Whitney (comparação intergrupos). Os resultados revelaram aumento significativo $(\mathrm{p}<0,05)$ no IGF1 (GCpós x GEsemana20 - $\Delta=54,29 \mathrm{ng} / \mathrm{ml}, \mathrm{p}=0,009$ ) e em todas as variáveis da força muscular (GEsemana4 x GEsemana20, $\Delta \%=39,23 \%$ e GEpré-teste $\mathrm{x}$ GEsemana20, $\Delta \%=56,08 \% ; \mathrm{p}<0,05)$. Desta forma, pode-se concluir que o treinamento de força muscular inferiu em aumento significativo desta variável e dos níveis séricos de IGF1 apenas na fase miogência.

\footnotetext{
Universidade Da Coruña, Facultade de Ciências do Deporte e a Educación Física Oleiros, A Coruña, Espanha

2 Universidade Castelo Branco. Programa de Pós-Graduação Stricto Sensu em Ciências da Motricidade Humana. Rio de Janeiro, RJ, Brasil
}

Correspondência / Correspondence

José Guilherme Fernandes Bertoni da Silva

Via Milly Mignone, n.13 - Milano - Itália. 20.153

E-mail: jgbertoni@virgilio.it / jgbertoni@fastwebnet.it

\author{
Palavras-chave: \\ Força Muscular. \\ Terapia por \\ Exercício. Desen- \\ volvimento \\ Muscular. Idoso. \\ Mulheres. IGF1. \\ Fase Neurogênica. \\ Fase Miogênica
}




\section{Abstract}

This study aimed to assess the effects of strength training on the levels of IGF1 and of muscle strength in neurogenic and miogenic phases of sedentary older. The sample consisted of 24 elderly, voluntary, randomly divided in two groups: the experimental group (EG, $\mathrm{n}=13 ; 65.62 \pm 5.36$ years) in the control group ( $\mathrm{CG}, \mathrm{n}=11 ; 71.45 \pm 5.72$ years). We used the protocol of maximum repetition (Baechle \& Groves, 1992) to evaluate muscle strength and maximum quimiluminescence - IMMULITE - DPC MED LAB for the IGF1. We used the Kruskal-Wallis (EG, three times) followed by multiple comparisons of Dunn; the Wilcoxon test (CG, in relation to two times) and the Mann-Whitney test (comparison inter-group). Results showed significant increase $(\mathrm{p}<0.05)$ in IGF1 (CGpost x EGweek20 $-\Delta=54.29 \mathrm{ng} / \mathrm{ml}, \mathrm{p}=0009$ ) and in all variables muscle strength (EGweek $4 \times$ EGweek20, $\Delta=39.23 \%$ and EGpre-test $x$ EGweek $20, \Delta \%=56.08 \%, \mathrm{p}<0.05)$. We concluded that training of muscular strength results in significant increase in this variable and serum levels of IGF1, only at the miogenic phase.

Key words: Muscle Strength. Exercise Therapy. Muscle Development. Aged. Women. IGF1. Neurogenic Phase. Miogenic Phase

\section{INTRODUÇÃO}

Segundo o Instituto Brasileiro de Geografia e Estatística, ${ }^{1}$ a expectativa de vida tem sofrido aumento progressivo. Há perspectivas de que a longevidade no Brasil, de 62,97 anos em 1980, passe a ser de 73,59 anos em 2050, quando o Brasil será considerado o sexto país a ter maior número de indivíduos idosos. Desta forma, a população brasileira, como ocorre nos demais países do mundo, encontra-se em um processo irreversível de envelhecimento demográfico.

O processo da senescência caracteriza-se pela perda progressiva das capacidades fisiológicas, com o declínio das funções de diversos órgãos e sistemas, dentre eles os sistemas endócrino e muscular. ${ }^{2-5}$

No sistema endócrino, pode-se encontrar uma redução de diversos hormônios, dentre eles, o hormônio de crescimento ( $\mathrm{GH}$ - growth hormone) e o fator de crescimento símile à insulina tipo um (IGF-1 insulin like growth factor). ${ }^{6}$ Sendo o IGF1 estimulado pelo $\mathrm{GH},{ }^{7}$ sua redução nos níveis séricos sanguíneos, especialmente em mulheres idosas, pode estar associada à diversas alterações, dentre elas à diminuição da massa magra è à atrofia muscular com redução da força. ${ }^{8-13}$

Em adição a esta alteração muscular decorrente do sistema endócrino, a senescência em si já repercute no declínio do sistema muscular, devido à redução no tamanho das fibras musculares (especialmente as do tipo II), fazendo com que ocorra gradual redução da massa muscular e da produção de força (sarcopenia) nos gerontes. ${ }^{5,10} \mathrm{Tal}$ fenômeno pode ser acelerado com a inatividade física, gerando maior nível de dependência funcional. ${ }^{14,15}$ 
Entretanto, os níveis de IGF-1 podem ser aumentados de forma endócrina, parácrina e autócrina, devido a inúmeros fatores, dentre eles o exercício físico. ${ }^{16}$ Dentre as diversas modalidades, o treinamento de força é o que apresenta maior correlação positiva, com aumentos séricos de IGF1. ${ }^{17}$ Portanto, a estimulação do IGF1 contribuirá de forma significativa para a hipertrofia e para o aumento do conteúdo de miofibrilas esqueléticas. ${ }^{18}$

O presente estudo teve por objetivo avaliar os efeitos do treinamento de força sobre os níveis basais de IGF1 e de força muscular nas fases neurogência e miogênica de idosas sedentárias.

\section{MATERIAIS E MÉTODOS}

Amostra

A amostra foi constituída de 24 idosas voluntárias, subdivididas, randomicamente, em dois grupos: grupo experimental (GE, $\mathrm{n}=13 ; 65,62 \pm 5,36$ anos) e grupo controle (GC, $\mathrm{n}=11 ; 71,45 \pm 5,72$ anos).

Os indivíduos envolvidos nesta pesquisa não podiam estar fazendo treinamento de força há pelo menos três meses. ${ }^{9}, 19$ Todas deveriam possuir autonomia funcional para realizar suas AVDs sem ajuda de terceiros ou de cuidadores e residir em seus domicílios na companhia de seus familiares.

Como critério de exclusão, foi considerado qualquer tipo de condição aguda ou crônica que pudesse ser um fator de impedimento ou comprometimento ao programa de treinamento de força, tais como cardiopatias, diabetes, hipertensão arterial e asma não controladas, quaisquer condições músculoesqueléticas que pudessem servir de fator interveniente à prática da atividade (osteoartrite, fratura recente, tendinite e uso de prótese), problemas neurológicos e uso de medicamentos que pudessem causar distúrbios da atenção e uso de terapia de reposição hormonal.

O universo foi constituído de 560 idosas. Após a primeira reunião para explanação da pesquisa, a mesma contou com um quantitativo de 118 gerontes voluntárias. Após o crivo dos critérios de inclusão e exclusão, pela anamnese e exame físico, a amostra findou em 56 mulheres, as quais foram distribuídas, por sorteio, no GE $(\mathrm{n}=28)$ e no GC $(\mathrm{n}=28)$. Entretanto, ao longo do experimento houve uma perda amostral (desistência, óbito, hospitalização e quedas) de 32 indivíduos, concluindo em 13 idosas no GE e 11 idosas no GC.

As participantes da pesquisa assinaram o termo de consentimento e os procedimentos experimentais foram executados dentro das normas éticas previstas na Resolução ${ }^{\circ}$ 196, de 10/10/1996, do Conselho Nacional de Saúde. O estudo teve seu projeto de pesquisa submetido e aprovado pelo Comitê de Ética em Pesquisa da Rede Euro-americana de Motricidade Humaan - REMH, RJ (protocolo n. 008/2008). ${ }^{20}$ 
Procedimentos

Avaliação da força muscular

Para a determinação da variável força muscular máxima, foi realizado o teste de 1RM pelo protocolo de Baechle e Groves, ${ }^{21}$ seguindo-se a orientação e a familiarização para a realização do mesmo, de acordo com as recomendações de Ploutz-Snyder e Giamis. ${ }^{22}$

Os exercícios utilizados na presente investigação foram: extensão de joelhos (EJ), flexão de joelho direito (FJD), flexão de joelho esquerdo (FJE), supino reto (SUP) e rosca tríceps (RT) no pulley.

O instrumento utilizado para essas aferições foi o aparelho aglomerado de musculação da marca Fitness Emporium Queens, H 500 (Brasil), com sistema de pesos em série.

Avaliação dos níveis séricos de IGF1

A fim de avaliar esta variável, as idosas foram submetidas a uma coleta de sangue (sistema fechado) a vácuo realizado por um laboratório em local previamente determinado. $\mathrm{O}$ método utilizado foi o quimiluminescência - IMMULITE - DPC MED LAB.

Os instrumentos necessários para esta coleta foram: algodão; álcool a 70\%; agulha descartável 0.80 × 25 - estéril; seringa descartável (5 ou $10 \mathrm{ml}$ ); "garrote" ou "torniquete"; tubo estéril sem anticoagulante, preferencialmente com gel separador.
Protocolo de intervenção

O GE foi submetido a uma intervenção (treinamento de força), por um período de 20 semanas, constituído de duas fases: $1^{a}$ : fase de adaptação (neurogênica) e $2^{\mathrm{a}}$ : fase de treinamento específico (miogênica). ${ }^{23}$

O programa de treinamento foi o do tipo alternado por segmento corporal, seguindo a ordem dos exercícios de supino na posição sentada, extensão de joelhos, puxada no pulley, flexão unilateral de joelhos, rosca bíceps simultânea, flexão parcial de tronco (abdominal) e rosca tríceps no pulley. A velocidade de execução foi de lenta a moderada, e a respiração utilizada, mais indicada nesta faixa etária, foi do tipo "passivo-eletiva”, ${ }^{24}$ que consiste na respiração contínua, expirando na fase concêntrica do movimento e inspirando na fase excêntrica.

A primeira fase teve a duração de quatro semanas, com uma frequência de treinamento de três vezes por semana, com intervalo entre 48 e 72 horas. Foram realizadas três séries de 13 repetições, com intervalos de aproximadamente 40 segundos, com o peso máximo de $50 \%$ de $1 \mathrm{RM}$.

A segunda fase teve a duração de 16 semanas, com uma frequência de treinamento de três vezes por semana, com intervalo entre 48 e 72 horas. Foram realizadas três séries de seis repetições, com intervalos de um a dois minutos entre as séries e entre os exercícios, com carga de 90 a $100 \%$ de 1 RM. 
Durante esta segunda fase, devido à adaptação do ganho de força, o controle da carga de treinamento foi feito utilizando-se o recurso proposto por Baechle e Groves, ${ }^{21}$ denominado de "regra de dois por dois" que consiste no ajuste da carga toda vez que o indivíduo realizar determinado exercício no limite superior (com dez repetições), por duas sessões seguidas.

Antes de cada sessão de treino, o GE foi submetido a 15 minutos de aquecimento com exercícios que envolvessem a mobilidade das principais articulações. Ao término de cada sessão, foi realizado relaxamento de cinco minutos com exercícios de alongamento em níveis submáximos. ${ }^{25}$

O GC manteve seus afazeres diários normais em todo o período do estudo. Este grupo se comprometeu a não realizar nenhuma atividade física sistematizada que envolvesse principalmente trabalho de força durante as 20 semanas de experimento até a realização do pós-teste.

\section{Tratamento estatístico}

O tratamento estatístico foi composto por análise descritiva, objetivando obter o perfil do conjunto de dados, através de medidas de localização (média e mediana), de dispersão (desvio-padrão - s, erro padrão da média, coeficiente de variação percentual - CV\%) e por análise inferencial através do teste de Shapiro-Wilk, para verificar a homogeneidade da amostra.
Foram utilizados: (a) o teste não-paramétrico de Kruskal-Wallis, para comparação do grupo experimental, em relação aos três momentos e, quando significativo o valor de $(\mathrm{H})$, foram empregadas as comparações múltiplas de Dunn; (b) o teste nãoparamétrico de Wilcoxon, para comparação do grupo controle, em relação aos dois momentos pré e pós e (c) o teste não-paramétrico Mann-Whitney, para comparação do grupo experimental e grupo controle no momento pré e no momento pós. ${ }^{25}$

\section{RESULTADOS}

A análise descritiva do GC demonstrou: (a) o IMC médio na classificação sobrepeso da OMS e (b) a média dos níveis séricos basais de IGF-1 dentro dos valores de referência para tal população, de acordo com o exame laboratorial ( $>64,00 \mathrm{a}<212,00 \mathrm{ng}$ ) $\mathrm{ml}$, quimiluminescência - IMMULITE DPC MED LAB). Na avaliação da homogeneidade do GC, observou-se normalidade $(p>0,05)$ apenas nas variáveis idade, massa corporal, estatura, IMC, IGF1 e EJ (tabela 1).

$\mathrm{Na}$ análise descritiva do GE, foi observado: (a) o IMC médio na classificação de sobrepeso da OMS e (b) a média dos níveis séricos basais de IGF-1 dentro dos valores de referência para esta população, de acordo com o exame laboratorial ( $>64,00$ a $<212,00 \mathrm{ng} / \mathrm{ml}$, quimiluminescência IMMULITE - DPC MED LAB). Na ava- 
liação da normalidade do GE, observouse que apenas as variáveis FJD, FJE, SUP e RT não apresentaram homogeneidade $(\mathrm{p}<0,05)$ (tabela 1).

Tabela 1: Estatística descritiva e inferencial de Shapiro-Wilk da amostra. 2008.

\begin{tabular}{ccccccc}
\hline & & Média & e & Mediana & $s$ & SW \\
\hline \multirow{2}{*}{ Massa corporal } & GC & 66,15 & 3,81 & 62,20 & 13,74 & 0,123 \\
& GE & 61,55 & 1,08 & 61,80 & 3,59 & 0,264 \\
Estatura & GC & 154,73 & 2,15 & 156,00 & 7,76 & 0,360 \\
IMC & GE & 156,65 & 1,76 & 158,00 & 5,83 & 0,534 \\
& GC & 27,40 & 1,17 & 26,30 & 4,23 & 0,206 \\
& GE & 25,35 & 0,86 & 24,70 & 2,84 & 0,258 \\
IGF-1 & GC & 97,04 & 6,70 & 95,60 & 22,21 & 0,038 \\
& GE & 130,77 & 9,42 & 130,00 & 33,96 & 0,334 \\
\multirow{2}{*}{ EJ } & GC & 16,82 & 1,69 & 15,00 & 5,60 & 0,211 \\
& GE & 21,54 & 1,64 & 20,00 & 5,91 & 0,386 \\
FJD & GC & 7,27 & 0,79 & 5,00 & 2,61 & 0,010 \\
& GE & 13,46 & 0,87 & 15,00 & 3,15 & 0,004 \\
FJE & GC & 7,27 & 0,79 & 5,00 & 2,61 & 0,010 \\
& GE & 13,46 & 0,87 & 15,00 & 3,15 & 0,004 \\
SUP & GC & 15,91 & 1,89 & 15,00 & 6,25 & 0,049 \\
& GE & 20,38 & 1,65 & 20,00 & 5,94 & 0,015 \\
RT & GC & 8,64 & 0,97 & 10,00 & 3,23 & 0,011 \\
& GE & 14,23 & 0,96 & 15,00 & 3,44 & 0,009 \\
\hline
\end{tabular}

GC: grupo controle; GE: grupo experimental; e: erro padrão; s: desvio-padrão; SW :ShapiroWilk; IMC: índice de massa corporal; IGF1: Fator de Crescimento Símile à Insulina tipo um; EJ: extensão de joelho; FJD: flexão de joelho direito; FJE: flexão de joelho esquerdo; SUP: supino; $\mathrm{RT}$ : rosca tríceps. As unidades de medida utilizadas foram: para a massa corporal $(\mathrm{Kg})$; para a estatura (centímetros); para o IMC (para IGF1 (ng/ml); para força (KG).

A análise estatística revelou aumentos significativos dos níveis de IGF1, pelo GE, na fase 2 (miogênica) em relação ao GC nesta fase $(\mathrm{p}=0,009)$. Nota-se, ainda, um decréscimo do IGF-1 do GE na fase 1, ao ser comparado com os níveis do pré-teste. Esses achados denotam que a variação absoluta ( $\Delta$ absoluto) do IGF-1 apresentou significância estatística favorável para o GE na fase miogênica (figura 1). Interessante ressaltar que os níveis de IGF1 encontrados em todas as fases, para ambos os grupos, apresentaram-se nos padrões de normalidade para a referida faixa etária, de acordo com o exame laboratorial. 


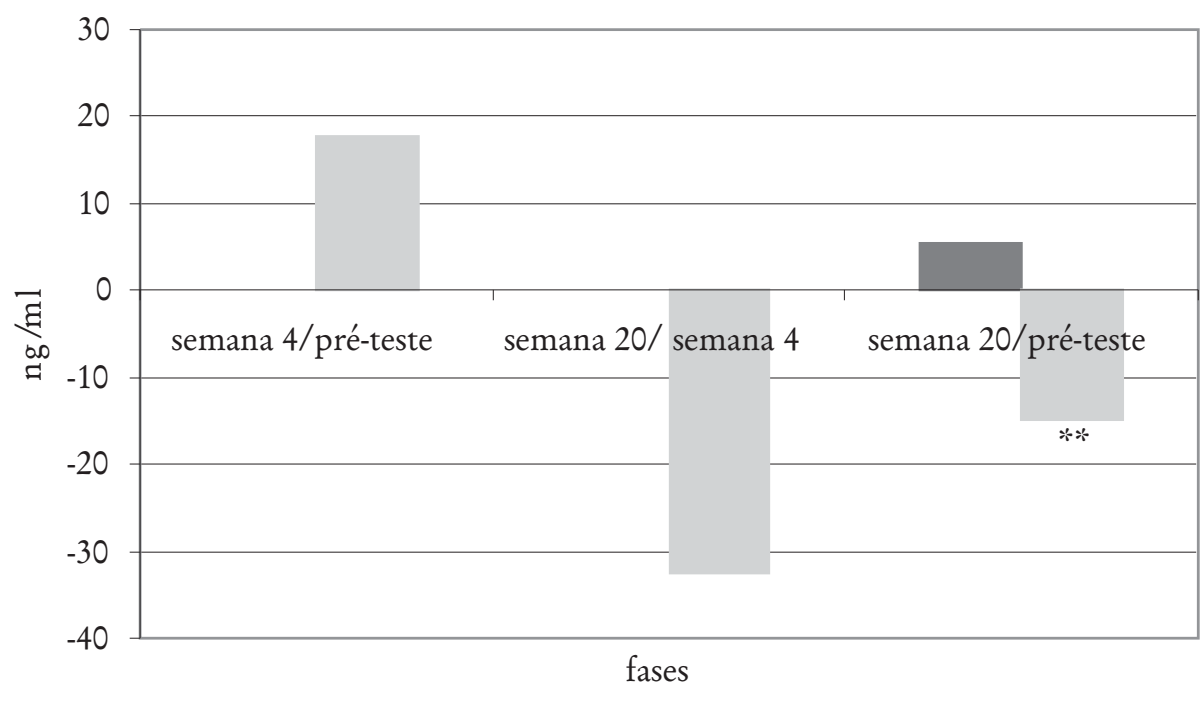

IGF1- GC IGF1- GE

**p $<0,05, \Delta$ IGF-1 (GCpós vs GEfase 2).

Figura 1: Comparação do $\Delta$ absoluto do IGF1 da amostra. 2008.

Os resultados nos níveis de força muscular do GE, na fase 2 (miogênica), revelaram ganhos significativos quando comparados ao pré-teste (valores de $\mathrm{p}: \mathrm{EJ}=$ 0,$0000 ; \mathrm{FJD}$ e $\mathrm{FJE}=0,0000 ; \mathrm{SUP}=$ 0,$0000 ; \mathrm{ET}=0,0000$ ) e à fase 1 (valores de $\mathrm{p}: \mathrm{EJ}=0,0008 ; \mathrm{FJD}$ e $\mathrm{FJE}=0,0031$; $\mathrm{SUP}=0,0005 ; \mathrm{ET}=0,0073)$. Essas respos- tas aos estímulos do treinamento de força demonstraram que a variação percentual $(\Delta \%)$ apresentou incrementos na força muscular favoráveis na 20a semana (figura 2). Pelo fato de o GC não ter realizado nenhuma atividade que envolvesse exercícios de força, esta variável fisiológica não foi analisada neste grupo. 


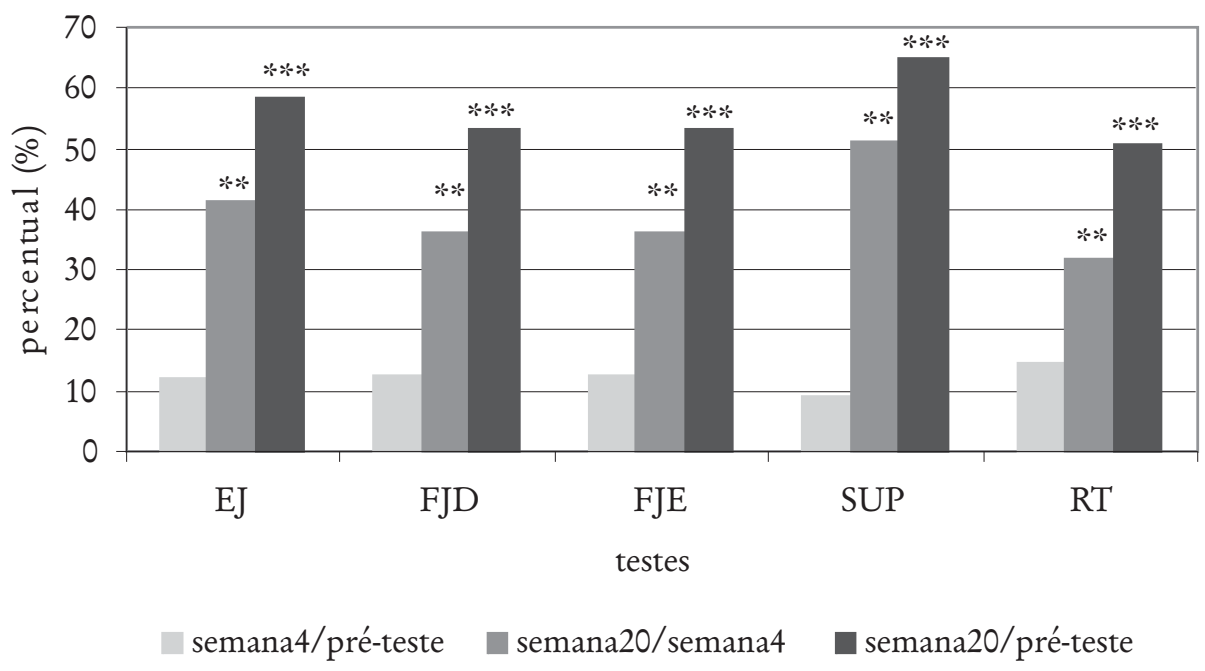

$*$ p $<0,05$ GE Sem4 (Fase 1) vs Sem20 (Fase 2)

$* * * \mathrm{p}<0,05$ GE pré-teste vs Sem20 (Fase 2)

Extensão de joelho (EJ); Flexão de joelho esquerdo (FJE); Flexão de joelho direito (FJD); Supino em posição sentada (SUP); Rosca tríceps no pulley (RT).

Figura 2: Comparação do $\Delta \%$ dos níveis de força Muscular do GE. 2008.

\section{DISCUSSÃO}

O presente estudo teve como hipótese o aumento significativo nos níveis séricos basais de IGF-1 na fase 2 (miogênica) do treinamento de força, quando comparados aos observados na fase 1 (neurogência) em mulheres idosas saudáveis. Com base nos resultados obtidos, o programa de treinamento aplicado proporcionou alterações significativas nos níveis séricos basais de IGF-1 na fase miogênica (fase 2), confirmando a hipótese levantada. Estes achados corroboram aqueles apresentados por Borst, De Hoyos e Garzarella, ${ }^{26}$ os quais revelaram elevações significativas no IGF-1 sérico basal após a $13^{\mathrm{a}}$ semana, de um total de 25 sema- nas de um programa de treinamento. Podese inferir que a plasticidade do sistema hormonal respondeu a exercícios de alta intensidade, longa duração e múltiplas séries, ${ }^{26-29}$ apontando a mesma tendência para o presente estudo, que teve duração de 20 semanas, utilizou cargas gradativas até alcançar 90\% de 1-RM e trabalhou com séries múltiplas (três séries).

Entretanto, nota-se na tabela 1 que houve redução dos níveis de IGF1 na fase 1. Isto pode ter ocorrido devido ao não-controle de alguns fatores, a saber: ingestão de álcool; nutrição; depressão de algumas proteínas; redução nas proteínas carreadoras do IGF (IGFBPs); má nutrição de micronu- 
trientes (magnésio, tiamina e zinco), as quais prejudicam a atividade deste hormônio, além de se associar a resistência periférica do hormônio de crescimento $(\mathrm{GH})$ e redução da expressão de IGF-1 no fígado. Desta forma, a dieta e a quantidade protéica são importantes para a manutenção dos níveis de IGF-1. ${ }^{30,31}$ Outras variáveis são apontadas e podem exercer influência na liberação e secreção deste hormônio no idoso: níveis de atividade física, ausência de doenças, padrões de sono, níveis de IGFBPs, receptores IGF-1 e atividade insulínica. ${ }^{28,30,32}$

Marx et al. ${ }^{33}$ também observaram resultados relevantes para a presente investigação. Nesta foram reportados aumentos significativos nas concentrações séricas basais de IGF-1 em mulheres sedentárias após seis meses de treinamento. Estes achados revelam que um programa de treinamento composto de alto volume e múltiplas séries possui magnitude maior quando comparado a uma série em circuito. Isto vem dar sustentação à afirmativa observada neste estudo, segundo a qual volume e intensidade de treinamento são variáveis importantes para adaptações crônicas de IGF-1.

Enquanto que alguns estudos têm encontrado efeitos positivos do treinamento de força muscular na liberação de GH e IGF1 em idosos, mostrando ainda associações entre os níveis hormonais, força, aumento de massa muscular e massa óssea, ${ }^{10,34}$ outras pesquisas contrapõem esses resultados e os da presente investigação, revelando não haver mudanças no IGF-1 durante ou imediatamente após o treinamento de força. ${ }^{11,35}$
O trabalho de Nindl et al. ${ }^{17}$ ressalta que o treinamento de força pode alterar o padrão temporal de liberação do GH durante a noite, e que o exercício resistido pode ocasionar alteração no sistema do IGF-1 e sua detecção na circulação. Este tipo de treinamento apresenta maior correlação positiva com aumentos séricos do IGF-1, dando sustentação aos resultados obtidos no estudo vigente. Estes níveis aumentam com o treinamento de força de forma endócrina, parácrina e autócrina. Portanto, além da liberação do IGF-1 pelo fígado, induzida pelo aumento de $\mathrm{GH}$, vários tecidos o produzem e ainda são estimulados a utilizá-lo como efeitos agudos do exercício. ${ }^{\text {17, 29, 30, } 36}$ Entretanto, a investigação de Aizawa, Hayashi e Mesak ${ }^{37}$ contrapõe tal informação, pois embora os autores tenham revelado uma correlação positiva do dehydroepiandrosterone sulfate (DHEAS) com o pico de torque muscular, nenhum resultado significativo foi encontrado com o IGF1.

A proposta do trabalho de Izquierdo $e t a l .^{38}$ foi examinar a eficácia de 11 semanas de treinamento de resistência (até a fadiga versus antes da fadiga), seguido de cinco semanas de treinamento de força nas mudanças fisiológicas de hormônios catabólicos e anabólicos na circulação basal. A amostra foi composta de 42 homens ativos, divididos, randomicamente, em grupo da fadiga (GF, $\mathrm{n}=14$ ), grupo sem fadiga (GSF, $\mathrm{n}=15$ ) e grupo controle (GC, $n=13$ ). As análise foram feitas em quatro momentos: (TO) préteste, (T1) após seis semanas de treinamento, (T2) após 11 semanas de treinamento e (T3) após 16 semanas de treinamento. Os 
grupos se apresentaram homogêneos quanto ao teste de $1 \mathrm{RM}$ e ao número de repetições alcançadas. Nos resultados, evidenciaram-se redução do IGF1 após o treinamento de força e elevação do IGFBP-3 no GF, enquanto o GSF resultou em redução do cortisol e elevação da testosterona. Esta redução do IGF1, em poucas semanas de treinamento de força, possui a mesma tendência daquela encontrada na primeira fase de treinamento deste estudo, exposta na figura 1.

O método de quimiluminescência - IMMULITE - DPCMED LAB utilizado nesta investigação é considerado, na atualidade, o gold standard para a avaliação sérica de IGF1, embora seja comum verificar em outras pesquisas relacionadas ao hormônio estudado o método radioimunoensaio. ${ }^{12,29,32,35}$ Tal informação deve ser ressaltada a fim de aumentar a credibilidade dos resultados expostos neste estudo.

A corrente pesquisa utilizou o teste de 1-RM para avaliar a força muscular do GE, pois alguns estudos demonstraram que este teste é um método eficaz, seguro e de alta reprodutibilidade para a quantificação da qualidade física força muscular, inclusive em idosos. ${ }^{27,28,39,40}$ Todavia, a pesquisa de Hortobágyi et al. ${ }^{41}$ utilizou o teste de 3-RM com a referida população, alegando que os idosos o toleram melhor devido à menor carga suportada, contrapondo o método utilizado por este estudo.

A pesquisa de Brooks et $\mathrm{al} .{ }^{42} \mathrm{com}$ homens diabéticos, além de utilizar o teste de $1 \mathrm{RM}$, também avaliou a histologia muscular atra- vés de biópsia. Com o mesmo tempo de treinamento de força utilizado na fase 2 do atual estudo (16 semanas), seus resultados revelaram: melhora significativa $(\mathrm{p}<0,001) \mathrm{da}$ qualidade de força muscular (grupo experimental: $28 \pm 3$ versus grupo controle: $-4 \pm 2$ ); aumento das fibras tipo I ( $\mathrm{p}=0,04$; grupo experimental: $860 \pm 252 \mu \mathrm{m} 2$ versus grupo cotrole: $-164 \pm 290 \mu \mathrm{m} 2)$ e das fibras tipo II $(\mathrm{p}=0,04$; grupo experimental: $720 \pm 285 \mu \mathrm{m} 2$ versus grupo controle: $-130 \pm 336 \mu \mathrm{m} 2$ ).

Este estudo ressaltou a importância do controle da intensidade de treinamento utilizando-se alta intensidade. Semelhantemente, esta afirmação foi levantada por Fatouros et al. ${ }^{43}$ Esses pesquisadores tiveram por objetivo determinar o efeito da intensidade de exercício na força na potência aeróbica e na mobilidade de idosos sedentários $(71,2 \pm 4$ anos) submetidos a 24 semanas de treinamento seguido de 48 semanas de destreino. A amostra foi dividida em: grupo controle (GC; $\mathrm{n}=14$ ); grupo de baixa intensidade (GBI; $n=18 ; 55 \%$ de $1 \mathrm{RM}$ ) e grupo de alta intensidade (GAI; $\mathrm{n}=20 ; 82 \%$ de $1 \mathrm{RM}$ ). Embora o GBI tenha melhorado $(\mathrm{p}<0.05)$ a força (42-66\%), a potência aeróbica (10\%) e a mobilidade (5-7\%), o GAI obteve melhoras mais significativas ( $\mathrm{p}<0.05$ ) na força (63-91\%), na potência aeróbica (17-25\%) e na mobilidade (9-14\%). Interessante ser ressaltado que, após 48 semanas de destreino, todos os ganhos do GBI foram abolidos, enquanto aqueles alcançados pelo GAI permaneceram.

Embora os mecanismos responsáveis pelo aumento da força muscular em idosos in- 
duzido pelo treinamento de força muscular ainda não sejam bem esclarecidos, há considerável aumento na eficiência (combustível) e na frequência máxima das unidades motoras. O combustível é garantido pelo mecanismo que sustenta as contrações em estado submáximo. Alguns investigadores defendem que a hipertrofia muscular nos gerontes é decorrente do treinamento de musculação e do nível sérico de IGF-1. ${ }^{44}$ Desta forma, o treinamento de força de alta intensidade é importante estimulador do GH e IGF-1 e os níveis deste último aumentam durante o exercício, em decorrência dos efeitos semelhantes à insulina do hormônio, ${ }^{6}$ sendo estes achados enrriquecedores para justificar os resultados da presente investigação. Portanto, os ganhos de força apresentados nesta pesquisa corroboram outros estudos. $27,33,41,45-47$

\section{CONCLUSÃO}

Conforme os resultados encontrados, o aumento significativo dos níveis séricos ba- sais de IGF-1 se deu na fase miogênica (Fase 2) no GE, o qual foi submetido ao treinamento de força muscular. Tal observação realça a hipótese do impacto positivo do fortalecimento muscular (de longa duração e alta intensidade) na melhora de IGF1 nos níveis basais. Entretanto, na fase neurogênica (Fase 1) foi analisada a redução desses níveis, possivelmente devido a algumas variáveis intervenientes, citadas no decorrer deste estudo.

Com relação aos níveis de força muscular, as idosas do GE apresentaram aumento significativo desta variável apenas na fase 2 , constatando-se que o ganho de força foi proveniente da adaptação miogênica e não da neurogênica.

Em suma, os resultados deste estudo permitem inferir que programas de treinamento de força para idosos com duração superior a quatro semanas são determinantes para reduzir a sarcopenia e, consequentemente, $\mathrm{o}$ impacto negativo da idade, pois a perda na força muscular e no desenvolvimento motor são fatores relevantes de prevalência para a incapacidade e a dependência. 


\section{REFERÊNCIAS}

1. Instituto Brasileiro de Geografia e Estatística. Bases de dados. [acesso em: 2006 jun 2]. < Disponível em: URL: http://www.ibge.gov.br >

2. Robergs RA, Roberts SO. Princípios fundamentais de fisiologia do exercício para a aptidão, desempenho e saúde. 1.ed. São Paulo: Phorte; 2002.

3. Marin RV, Matsudo S, Matsudo V, Andrade E, Braggion G. Acréscimo de 1 $\mathrm{Kg}$ aos exercícios praticados por mulheres acima de 50 anos: impacto na aptidão física e capacidade funcional. Revista brasileira de ciência \& movimento 2003; 11(1): 53-8.

4. Balagopal P, Rooyackers OE, Adey D.B, Ades PA, Nair KS. Effects of aging on in vivo synthesis of skeletal muscle myosin heavy-chain and sarcoplasmic protein in humans. J Appl Physiol 1997; 273(4): E790-E800.

5. Hunter GR, Wetzstein CJ, Mclafferty Junior CL, Zucckerman PA, Landers KA, Bamman MM. High-resistance versus variable-resistence training in older adults. Med Sci Sports Exerc 2001; 33(10): 1759-64.

6. Eliakim A, Oh Y, Cooper DM. Effect of single wrist exercise on fibroblast growth factor-2, insulin-like growth factor, and growth hormone. J Appl Physiol 2000; 279(2): R548-53.

7. Ameredes BT, Watchko JF, Daood MJ, Donahoe MP, Rogers R. Growth hormone improves body mass recovery with refeeding after chronic under nutritioninduced muscle atrophy in aging male. J Nutr 1999; 129: 2264-70.

8. Fernholm, R, Bramnert M, Hägg E, Hilding A, Baylink DJ, Mohan S, Thorén
M. Growth hormone Replacement Therapy Improves Body Composition and Increases Bone Metabolism in Elderly Patients with Pituitary Disease. J Clin Endocrinol Metab 2000; 85(11):4104-11.

9. Lemmer JT, Hurlut DE, Martel GF, Tracy BL, Ivey FM, Metter EJ, Fozard JL, Hurley BF. Age and gender responses to strength training and detraining. Med Sci Sports Exerc 2000; 32(8): 1505-12.

10. Liu W, Thomas SG, Asa SL, GonzalezCadavid N, Bhasin S, Ezzat S. Myostatin is a skeletal muscle target of growth hormone anabolic action. J Clin Endocrinol Metab 2003; 88: 5490-6.

11. Häkkinen K, Pakarinen A, Kraemer WJ, Häkkinen A, Valkeinen H, Alen M.

Selective muscle hypertrophy, changes in EMG and force, and serum hormones during strength training in older women. J Appl Physiol 2001; 91(2): 569-80.

12. Thomas SC, Esposito JG, Ezzat S. Exercise Training benefits Growth hormone (GH) deficient adults in the absence or presence of GH treatment. J Clin Endocrinol Metab 2003; 88(12): 5734-8.

13. Shalet SM. Growth hormone outgrows growth. Clinical Endocrinology 2004; 61(1): 1-9.

14. Berlezi EM, Rosa PV, Souza ACA, Schneider RH. Comparação antropométrica e do nível de aptidão física de mulheres acima de 60 anos praticantes de atividade física regular e não praticantes. Revista brasileira de geriatria e gerontologia 2006; 9(3).

15. Pereira FF, Monteiro N, Vale RGS, Gomes ALM, Dantas EHM. Efecto del entrenamiento de fuerza sobre la autonomía funcional en mujeres mayores 
sanas. Revista Especial Geriatria Gerontologia 2007; 42(6): 319-24.

16. Gomes RJ, Luciano E, Caetano FH. Influência do treinamento físico sobre o fator de crescimento insulino-símile (IGF1) em ratos machos wistar. Revista Brasileira Atividade Física e Saúde 2001; 6(1): 25-30.

17. Nindl BC, Kraemer WJ, Marx JO, Arciero PJ, Dohi K, Kellog MK, Loomis GA. Overnight responses of the circulating IGF1 system after acute, heavy-resistance exercise. J Appl Physiol 2001; 90(4): 1319-26.

18. Trappe S, Godard M, Gallagher P, Carrol C, Rowden G, Porter D. Resistance training improves single muscle fiber contractile function in older women. J Appl Physiol 2001; 281(2): C398-406.

19. Raso V, Matsudo SM, Matsudo VKR. A força muscular de mulheres idosas decresce após oito semanas de interrupção de um programa de exercícios com pesos livres. Revista Brasileira de Medicina do Esporte 2001; 7(6).

20. Baechle TR, Groves BR. Weight training: steps to success. Champaign: Human Kinetics; 1992.

21. Ploutz-Snyder LL, Giamis EL. Orientation and familiarization to $1 \mathrm{RM}$ strength testing in old and young women. J Strength Cond Res 2001; 15(4): 519-23.

22. Dantas EHM. A prática da preparação física. 5·ed. Rio de Janeiro: Shape; 2003.

23. Westcott W, Baechle T. Treinamento de força para a terceira idade. 1. ed., São Paulo: Manole; 2001.

24. Dantas EHM. Flexibilidade: Alongamento e Flexionamento. 4. ed. Rio de Janeiro: Shape; 1999.

25. Thomas JR, Nelson JK, Silverman S. Métodos de pesquisa em atividade física. 5. ed. Porto Alegre: Artmed; 2005.
26. Borst SE, De Hoyos DV, Garzarella L. Effects of resistance training on insulinlike growth factor-I and IGF binding proteins. Med Sci Sports Exerc 2001; (33): 648-53.

27. Kalapotharakos VI, Michalopoulos SP, Goldolias G, Gourgoulis V. Effects of heavy and moderate resistance training on functional performance in older adults. J Strength Cond Res 2005; 19(3): 652-7.

28. Rubin MR, Kraemer WJ, Maresh CM, Volek JS, Ratamess NA, Vanheest JL, Silvestre R, French DN, Sharman MJ, Judelson DA, Gómez AL, Vescovi JD, Hymer WC. High-affinity growth hormone binding protein and acute heavy resistance exercise. Med Sci Sports Exerc 2005; 37(3): 395-403.

29. Ruiz-Torres A, Kirzner MSM. Ageing and Longevity are related to Grrowth Hormone/ Insulin - like Growth Factor 1 secretion. Gerontology 2002; 48(6): 401-7.

30. Arvat E, Broglio F, Ghigo E. Insulin-like growth factor I: implications in aging. Drugs Aging 2000; 16(1): 29-40.

31. Norat T, Dossus L, Rinaldi S, Overvad K, Gronbæk K, Tjonneland A, et al. Diet, serum insulin-like growth factor-I and IGF-binding protein-3 in European women. Eur J Clin Nutr 2007; 61: 91-8.

32. Capolla AR, Bandeen-Roche K, Wand GS, Volpato S, Fried LP. Association of IGF-1 Levels with Muscle Strength and Mobility in Older Women. J Clin Endocrinol Metab 2001; 86(9): 4139-45.

33. Marx JO, Ratamess NA, Nindl BC, Gotshalk LA, Volek JS, Dohi K, Bush JA, Gómez AL, Mazzetti SA, Fleck SJ, Häkkinen K, Newton RU, Kraemer WJ. Low-volume circuit versus high-volume periodized resistance training in women. Med Sci Sports Exerc 2001; 33: 635-43. 
34. Cress ME, Buchner DM, Prohaska T, Rimmer J, Brown M, Macera C, Depietro L, Chodzko-Zajko W. Physical Activity Programs and Behavior Counseling in Older Adult Populations. Official Journal of the American College of Sports Medicine. Med SciSports Exerc 2004; p.1997-2003.

35. Walker KS, Kambadur R, Mridula S, Smith HK. Resistance Training Alters Plasma Myostatin but not IGF-1 in Healthy Men. Med Sci Sports Exerc 2004; 36(5): 787-93.

36. Lambert C, Bopp M, Johnson L, Sullivan D. Resistence Training and Testosterone Replacement Induced Xhanges in Body Composition, Free Testosterone, IGF1 and IGFBP-3 in the Fraily Elderly. Journal of exercise physiology online 2007; 10 (1): 48-56.

37. Aizawa K, Hayashi K, Mesak N. Relationship of Muscle Strength with Dehydroepiandrosterone Sulfate (DHEAS), Testosterone and Insulin-like Growth FactorI In Male and Female Athlete. Adv.Exerc.Sports Physiol 2006; 12(1): 29-34.

38. Izquierdo M, Ibanez J, Gonza'LezBadillo JJ, Ha“Kkinen K, Ratamess NA, Kraemer WJ, French DN, Eslava J, Altadill A, Asiain X, Gorostiaga EM. Differential effects of trength training leading to failure versus not to failure on hormonal responses, strength, and muscle power gains. J Appl Physiol 2006; 100: 1647-56.

39. Fish DE, Krabak BJ, Johnson-Greene D, Delateur BJ. Optimal resistance training: Comparison of De Lorme with Oxford techniques. American Journal Physical Medicine \& Rehabilitation 2003; 82: 903-9.

40. Goto KM, Nagasawa M, Yanagisawa O, Kisuka T, Ishii N, Takamatsu K. Muscular

Recebido: $24 / 4 / 2008$

Aprovado: 20/11/2008 adaptations to combinations of high- and low- intensive resistense exercises. J Strength Cond Res 2004; 18: 730-7.

41. Hortobágyi T, Money J, Zheng D, Dudek R, Fraser D, Dohin L. Muscle adaptations to 7 days of exercise in young and older humans: eccentric overload versus standard resistive training. J Aging Phys Act 2002; 10: 290-305.

42. Brooks N, Layne JE, Gordon PL, Roubenoff R, Nelson ME, CastanedaSceppa C. Strength training improves muscle quality and insulin sensitivity in Hispanic older adults with type 2 diabetes. Int J Med Sci 2007; 4(1): 19-27.

43. Fatouros IG, Kambas A, Katrabasas I, Nikolaidis K, Chatzinikolaou A, Leontsini D, Taxildaris K. Strength training and detraining effects on muscular strength, anaerobic power, and mobility of inactive older men are intensity dependent. Br J Sports Med 2005; 39 (10): 776-80.

44. Hurley BF, Roth SM. Strength training in the elderly: effects on risk factors for agerelated diseases. Sports Med 2000; 30(4): 249-68.

45. Katula JA, Sipe M, Rejeski WJ, Focht BC. Strength training in older adults: an empowering intervention. Med Sci Sports Exerc 2006; 38(1): 106-11.

46. Vincent KR, Braith RW. Resistance exercise and bone turnover in elderly men and women. Med Sci Sports Exerc 2002; 34(1): 17-23.

47. Whitehurst MA, Johnson BL, Parker CM, Brown LE, Ford AM. The benefits of a functional exercise circuit for older adults. J Strength Cond Res 2005; 19: 647-51.

48. Brasil. Ministério da Saúde. Conselho Nacional de Saúde. Resolução 196/96. Brasília: CNS, 1996 